\title{
Effect of Battle Rope exercise on certain physical variables and numerical level of discus throw for female students
}

\section{Faten abo el sood emam}

\section{Introduction.}

There is no doubt that sports training has general rules, as what researchers have followed in developing sports performance in appropriate and proper contexts, where everyone is currently seeking to achieve sporting achievements through keeping up with the latest training methods used to develop the level of sports performance.

Studying the motor performance of a person in various sporting activities has become one of the issues that concern workers in the field of teaching and training the motor skills related to various sporting activities in order to study the factors affecting human performance in a direct or indirect way, to reach instructions by which to direct the process of education and training and improve motor performance to achieve the best results during competitions (14: 127).

track and Field competitions are considered one of the sports that depend on physical abilities, which directly affects the numerical level of the various competitions, such as running, jumping and throwing competitions. (13:1)

Throwing and pushing competitions are considered to be one of the branches of field and track competitions, goal of throwing competitions is determined to reach the farthest distance possible for the tool from exploiting all the potential forces to get this distance, and throwing competitions are characterized by their performance in rapid strength, speed, mobility, and agility that affect skill performance through the speed of the launch of the tool from the competitor's hand through transmission The kinetic speed from the rider's body to the instrument. $(7: 24)$

The Discus Throwing competition requires a set of physical characteristics such as (Muscular strength - ability - kinetic speed - flexibility - agility - balance - compatibility). (13: 2) 
The researcher believes that for the effective performance of the discus throw competition during the technical stages of performance that start from grabbing the instrument and the performance of the invasion and rotation and ends to throw and balance.

Modern methods are used in training programs to improve the level of performance to develop (time - distance - height), as they are considered true indicators to determine the level of performance in the training process, so the use of modern tools and devices and their use in an innovative way helps to develop efficient physical and skill performance that is reflected in the level of skill performance.

Fontaine \& Schmidt. (2015) point out that the continuous and increasing emphasis towards achieving sport achievement has led scientists to search for training methods that have positive effects on performance, and rope training exercise is one of these methods that has attracted attention in recent times.

Colin Mc-Auslan. (2013) notes that the battel rope, sometimes called the heavy rope, is one of the latest fitness trends used in gyms around the world, as it is used as a training tool for athletes in general and for athletes in particular, and can be used through it. Moving muscles in new ways and means. (4:112)

Calatayud, et al. (2015) The use of a battel rope is a dynamic, everchanging form of movement, where ropes can be flogged, beaten, or dragged, but among the most popular exercises are wavy movements that cause ropes to move in the form of waves. (2: 2854)

Colin Mc-Auslan. (2013) adds that the battel rope is often seen as an instrument used only for the upper end of the body, and this is not correct as it is possible to grow the center muscles in addition to the upper end where various muscle groups such as jumping, stabbing and changing positions can be involved The body during performance. (4: 113)

From the previous introduction the researcher noted the importance of using modern exercise tools that contribute to developing physical capabilities. Research Aim. Aim of the research:

1- The research aims to identify the effect of Battle Rope exercise on certain physical variables of discus throw for female students.

2- The research aims to identify the effect of Battle Rope exercise on numerical level of discus throw for female students. 


\section{Research hypotheses:}

3- There are statistically significant differences between the pre and post measurements of control group on some physical variables and numerical level of discus throw for the benefit of the post measurements.

4- There are statistically significant differences between the pre and post measurements of experimental group on physical variables and numerical level of discus throw for the benefit of the post measurements.

5- There are statistically significant differences between the post measurements of control group and experimental group on physical variables and numerical level of discus throw for the benefit of experimental group.

\section{Research terms:}

\section{BATTLE ROPE:}

are used for fitness training to increase full body strength and conditioning. It was designed by John Brookfield, who developed the battle rope exercise around 2006 .

\section{Search procedures:}

\section{Research approach:}

The experimental approach used two groups control and experimental group with the pre and post measurement standard design.

\section{Research community:}

The research community includes female physical education students $3^{\text {st }}$ stage university year 2019/2020.

\section{Research Sample:}

The subjects for this experiment from Faculty of Physical Education track \& field 3st stage students, $(n=35)$ divided to (15) students for experimental group, (15) students for control group and (5) students for the pilot sample. 
Table (1)

Mean \pm SD and skewness for recherché variables (age, height, weight, muscle strength, power, agility, coordination \& discus throw numerical level)

\begin{tabular}{l|c|c|c|c}
\hline \multicolumn{1}{c|}{ Variables } & measuring unit & Mean & SD & skewness \\
\hline \hline Age & years & $\mathbf{2 0 . 6}$ & 0.5 & 0.95 \\
\hline Height & $\mathrm{Cm}$ & $\mathbf{1 6 5 . 8}$ & 2.55 & $\mathbf{1 . 4 5}$ \\
\hline Weight & $\mathrm{kg}$ & $\mathbf{6 0 . 4}$ & $\mathbf{2 . 8 5}$ & $\mathbf{0 . 8 9}$ \\
\hline $\begin{array}{l}\text { Arm strength test (Bench } \\
\text { Press) }\end{array}$ & $\mathrm{kg}$ & 29.75 & 2.25 & $\mathbf{1 . 0 9}$ \\
\hline $\begin{array}{l}\text { Leg strength test (Leg } \\
\text { Press) }\end{array}$ & $\mathrm{kg}$ & $\mathbf{5 6 . 7 5}$ & $\mathbf{5 . 5 0}$ & $\mathbf{1 . 1 2}$ \\
\hline Starting long jump test & $\mathrm{M}$ & $\mathbf{2 . 5 0}$ & $\mathbf{0 . 7 5}$ & $\mathbf{0 . 8 5}$ \\
\hline Medicine ball throw test & $\mathrm{M}$ & $\mathbf{1 1 . 7 2}$ & $\mathbf{2 . 8 5}$ & $\mathbf{1 . 0 3}$ \\
\hline Hexagonal test & Sec & $\mathbf{1 9 . 8 0}$ & $\mathbf{4 . 5 0}$ & $\mathbf{2 . 2 1}$ \\
\hline Hand-Eye Test & $\mathrm{N}$ & $\mathbf{2 0 . 0 0}$ & $\mathbf{3 . 0 0}$ & $\mathbf{1 . 9 0}$ \\
\hline discus throw numerical level & $\mathrm{M}$ & $\mathbf{1 1 . 8 5}$ & $\mathbf{2 . 7 9}$ & $\mathbf{2 . 1 0}$ \\
\hline \hline
\end{tabular}

Table 1 shows: observed in (Age, height, weight, arm strength, leg strength, arm and leg power, agility, coordination, discus throw numerical level), ranging between $(0.07-2.44)( \pm 3)$ indicating that the sample combined represents a moderate society in these variables.

\section{Material and Methods:}

\section{Measurements tools:}

* Restameer /for measuring length $(\mathrm{cm})$.

* Medical balance/ to measure the weight of the nearest half a kilogram $(\mathrm{kg})$.

* Barbell and weights

* Leg Press machine.

* Tape measure/ (cm).

* Cones.

* Battel ropes.

\section{Research Tests:}

1- Physical variables Tests.

- Arm strength (Bench Press test).

- Leg strength (Leg Press test).

- Arm power (Starting long jump test).

- Leg power (Medicine ball throw test).

- Agility (Hexagonal test).

- Coordination (Hand-Eye Test). 


\section{2- discus throw numerical level.}

\section{Suggested exercises:}

\section{1- Aim of exercises.}

Improve physical variables and numerical level of discus throw competition for the research sample.

\section{2- Foundations of the development of training:}

- Attention to warm up and prepare the body for training.

- Suitable battel rope exercises for the research sample.

- Giving consideration of individual differences between students.

- Progressive of exercises from easier to harder and from simple to complex.

- Variety of exercise inside the training units.

\section{3- Exercises training program:}

- Experiment Duration (8) weeks.

- Number of units (24) units (3) units per week.

- The time required to apply the exercises (25) minutes battel rope exercise during the unit.

- Training unit time (75-90) minutes divided as follows:

- Warm up $(15 \mathrm{~m})$.

- Specific event workout $(60 \mathrm{~m})$ divided into:

- Technic to teach and improve the discus throw performance.

- Battel rope exercise for the development of body movements.

- The Cool-down (5m).

\section{Research procedure: Survey Study:}

The Survey study includes (5) students from the research community and outside the experimental research sample from $1 / 10 / 2019$ to $3 / 10 / 2019$ to achieve the following objectives.

1- Identify the suitability of battel rope exercise for students.

2- Identify the selection of tests in terms of the order and time of application of each test commensurate with the students. 


\section{Pre measurement:}

The measurements were applied from 5/10/2019 the measurements and tests included the following variables: (Arm strength test (Bench Press) - Leg strength test (Leg Press) - Starting long jump test - Medicine ball throw test Hexagonal test - Hand-Eye Test - discus throw numerical level.

\section{Research implementation:}

The research experiment was implemented at faculty of physical education for girls, from 6/10/2019 to 20/11/2019 for (8 weeks), (24) units, (2) days per week.

\section{Post measurement:}

Post-measurements for all research variables were applied from 22/11/2019.

\section{Statistical analysis}

All statistical analyses calculated by the SPSS statistical program. The results reported as means and standard deviations (SD).

- Arithmetic Mean.

- Standard Deviation .

- Skewness.

- Median.

- Paired Samples T-Test

- Percentage of improvement rates. 


\section{Results.}

Table (2)

Significance of differences between pre and post measurement of physical variables and numerical level of discus throw for control group

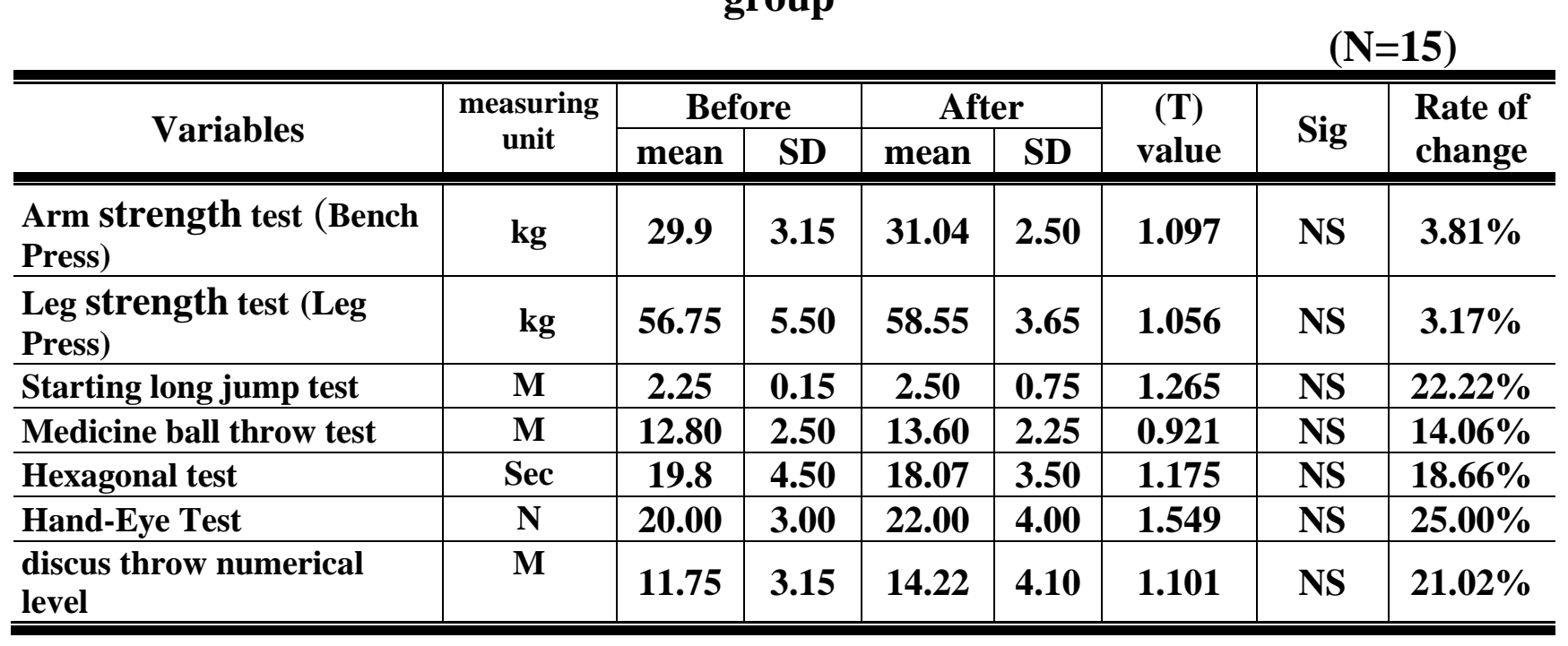

(T) Value of $T$ is the table at a significant level $(0.05)=1.753$

Table (2) shows: non-Significant Difference between the pre and post measurements of control group on (leg strength, arm and leg power, agility, coordination, discus throw numerical level) \& numerical level of discus throw. The rate of change was between $(3.18 \%-25.00 \%)$. 
Table (3)

Significance of differences between the pre and post measurement of physical variables and numerical level of discus throw for experimental group)

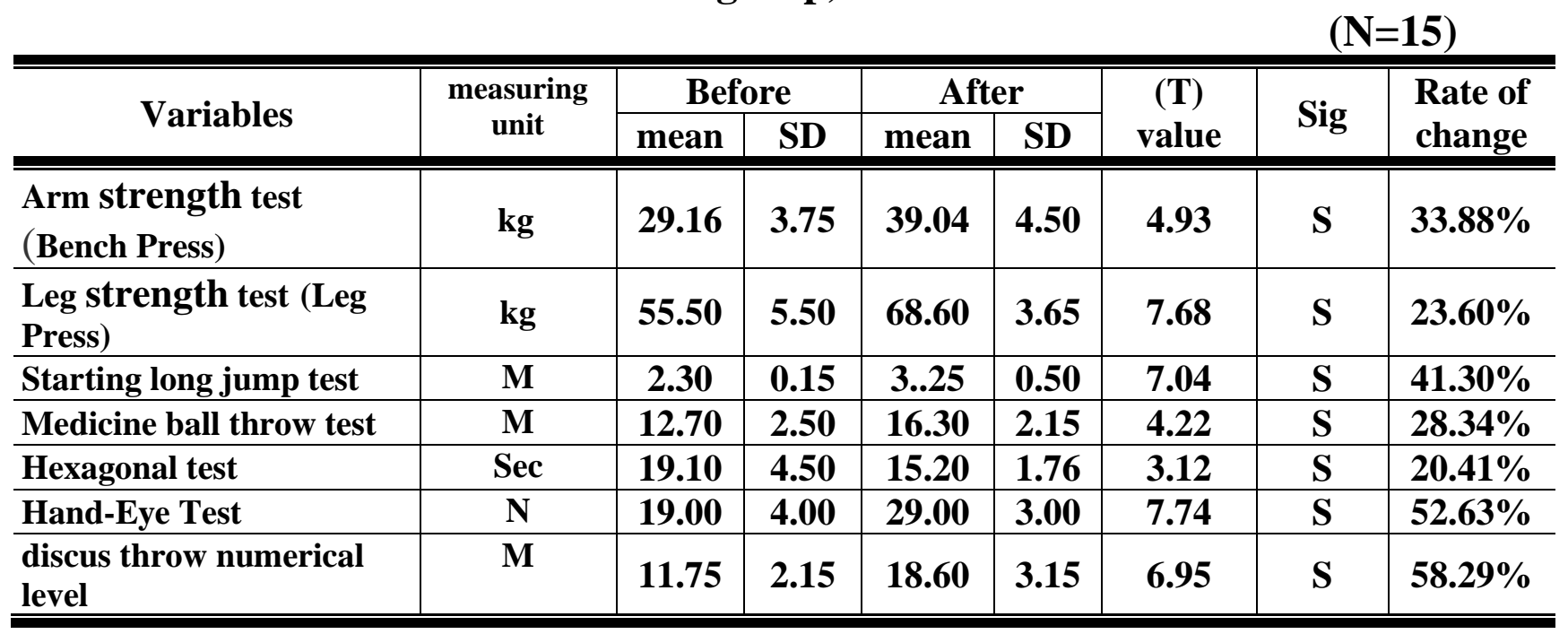

(T) Value of $T$ is the table at a significant level $(0.05)=1.753$

Table (3) shows: Significant Difference between the pre and post measurements of experimental group on (leg strength, arm and leg power, agility, coordination, discus throw numerical level) \& numerical level of discus throw. The rate of change was between $(20.41 \%-58.29 \%)$. 
Table (4)

Significance of differences between the control and experimental group on physical variables and numerical level of discus throw

\begin{tabular}{|c|c|c|c|c|c|c|c|}
\hline \multirow{2}{*}{ Variables } & \multirow{2}{*}{$\begin{array}{c}\text { measuring } \\
\text { unit }\end{array}$} & \multicolumn{2}{|c|}{ control } & \multicolumn{2}{|c|}{ experimental } & \multirow{2}{*}{$\begin{array}{l}\text { (T) } \\
\text { value }\end{array}$} & \multirow{2}{*}{ Sig } \\
\hline & & mean & SD & mean & SD & & \\
\hline $\begin{array}{l}\text { Arm strength test } \\
\text { (Bench Press) }\end{array}$ & kg & 31.04 & 2.50 & 39.04 & 3.50 & 10.18 & $S$ \\
\hline $\begin{array}{l}\text { Leg strength test (Leg } \\
\text { Press) }\end{array}$ & kg & 58.55 & 3.65 & 68.60 & 3.65 & 10.66 & $\mathrm{~S}$ \\
\hline Starting long jump test & M & 2.50 & 0.75 & 3.25 & 0.50 & 4.55 & $\mathrm{~S}$ \\
\hline $\begin{array}{l}\text { Medicine ball throw } \\
\text { test }\end{array}$ & M & 13.60 & 2.25 & 16.30 & 2.15 & 4.75 & $\mathrm{~S}$ \\
\hline Hexagonal test & Sec & 18.07 & 3.50 & 15.20 & 1.76 & 4.01 & $\mathrm{~S}$ \\
\hline Hand-Eye Test & $\mathbf{N}$ & 22.00 & 4.00 & 29.00 & 3.00 & 7.66 & $\mathrm{~S}$ \\
\hline $\begin{array}{l}\text { discus throw numerical } \\
\text { level }\end{array}$ & M & 13.22 & 4.10 & 18.60 & 3.15 & 5.69 & 5 \\
\hline
\end{tabular}

(T) Value of $T$ is the table at a significant level $(0.05)=1.697$

Table (4) shows: Significant Difference between the control and experimental measurements on (leg strength, arm and leg power, agility, coordination, discus throw numerical level) \& numerical level of discus throw for the favor of experimental group.

\section{Discussion:}

Table (2) shows: non-Significant Difference between the pre and post measurements of control group on (leg strength, arm and leg power, agility, coordination, discus throw numerical level) \& numerical level of discus throw. The rate of change was between $(3.18 \%-25.00 \%)$.

The Researchers attributed these results to Practice the control group to the traditional method of teaching and training for discus throw competition. Furthermore, the traditional method does not Containing exercises that help to develop the elements of fitness for the discus throw competition, but depends on a set of traditional exercises and repeat the performance discus throw only. Therefore, there is a deficiency in the rates of development of specific physical fitness and therefore inadequate in the required level of performance and numerical performance of students. 
Table (3) shows: Significant Difference between the pre and post measurements of experimental group on (leg strength, arm and leg power, agility, coordination, discus throw numerical level) \& numerical level of discus throw. The rate of change was between $(20.41 \%-58.29 \%)$.

Table (4) shows: Significant Difference between the control and experimental measurements on (leg strength, arm and leg power, agility, coordination, discus throw numerical level) \& numerical level of discus throw for the favor of experimental group.

The Researcher attributed these results to Battel rope exercise that helps to developing body dynamic movement in an effective way, through variety in the exercises of battel ropes.

Also Battle rope exercises enhanced explosive power of upper and lower limp and which positively effects on specific physical variables and the level of discus throw numerical achievement.

Colin McAuslan (2013) notes that the battel rope, sometimes called the heavy rope, is one of the latest fitness trends used in gyms around the world, as it is used as a training tool for athletes in general and for athletes in particular, and can be used through it. Moving muscles in new ways and means. (6: 112)

Calatayud, et al. (2015) The use of a battel rope is a dynamic, everchanging form of movement, where ropes can be flogged, beaten, or dragged, but among the most popular exercises are wavy movements that cause ropes to move in the form of waves. (4: 2854)

Colin McAuslan (2013) adds that the battel rope is often seen as an instrument used only for the upper end of the body, and this is not correct as it is possible to grow the center muscles in addition to the upper end where various muscle groups such as jumping, stabbing and changing positions can be involved The body during performance. (6: 113)

From the previous results, the researcher tried to shed light on the importance of using modern training tools because of their positive impact on physical capabilities, as track and field competitions depend on physical capabilities because of their positive impact on the level of achievement. 


\section{Conclusion:}

1- There is non-Significant Difference between the pre and post measurements of control group on (leg strength, arm and leg power, agility, coordination, discus throw numerical level) \& numerical level of discus throw. The rate of change was between $(3.18 \%-25.00 \%)$.

2- There is Significant Difference between the pre and post measurements of experimental group on (leg strength, arm and leg power, agility, coordination, discus throw numerical level) \& numerical level of discus throw. The rate of change was between $(20.41 \%-58.29 \%)$.

3-There is Significant Difference between the control and experimental measurements on (leg strength, arm and leg power, agility, coordination, discus throw numerical level) \& numerical level of discus throw for the favor of experimental group.

\section{4- Recommendations:}

1- The interest in using modern fitness training, such as Battel rope exercises for their positive effects on physical fitness, especially for students.

2- Conducting such a study on different track and field events.

3- Conducting such a study on other kinds of samples. 


\section{References:}

1. Brewer W, Kovacs R, Hogan K, Felder D, Mitchell H. (2017). Metabolic Responses to a Battling Rope Protocol Performed in the Seated or Stance Positions, J Strength Cond Res;32(12):3319-3325.

2. Calatayud, J, Martin, F, Colado, JC, Benı'tez, JC, Jakobsen, MD, and Andersen, LL. (2015). Muscle activity during unilateral vs. bilateral battle rope exercises. J Strength Cond Res 29: 2854-2859.

3. Chen, WH, Wu, HJ, Lo, SL, Chen, H, Yang, WW, Huang, CF , and Liu, C. (2017). Eight-week battle rope training improves multiple physical fitness dimensions and shooting accuracy in collegiate basketball players. J Strength Cond Res, Volume 32 - Issue 10 - p 2715-2724.

4. Colin McAuslan (2013). Physiological Responses to a Battling Rope High Intensity Interval Training Protocol, electronic Theses and Dissertations. 4853, University of Windsor.

5. Fountaine, CJ and Schmidt, BJ. (2015). Metabolic cost of rope training. J. Strength Cond Res 29: 889-893.

6. Gerry A. Carr (1991): "Fundamentals of Track and Field", University of Victoria. 110-117

7. Horvatin-Fučkar, M., Oreb, G., Tkalčić, S., \& Vlašić, J. (2005). Relations Between Rhythmic Abilities and Success in Artistic and Rhythmic Gymnastics. In (Ed.) Proceedings book of Fifth Journess Internationales de L' AFRAGA. (pp. 43-47).

8. Hutchinson, M.R., Tremain, L., Christiansen, J., \& Beitzel, J. (1998). Improving leaping ability in elite rhythmic gymnasts. Medicine and Science in Sport and Exercise, 30(10), 1543-1547.

9. Kramer, K., Kruchten, B., Hahn, C., Janot, J., Fleck, S., \& Braun, S. (2015) The effects of kettlebells versus battle ropes on upper and lower body anaerobic power in recreationally active college students. Journal of Undergraduate Kinesiology Research.

10. Marín, P. J., García-Gutiérrez, M. T., Da Silva-Grigoletto, M. E., \& Hazell, T. J. (2015). The addition of synchronous whole-body vibration to battling rope exercise increases skeletal muscle activity. J Musculoskelet Neuronal Interact, 15(3), 240-248.

11. McAuslan, C. (2013). Physiological Responses to a Battling Rope High Intensity Interval Training Protocol. University of Windsor.

12. Mikhail Shestakov (2014): Modeling and simulation in discus throw, Publisher: Routledge, Editors: Youlian Hong, pp.618.

13. Mostafa Atwaa, Mohammed Gamal (2019): Better Way to Throw the Discus? https://trackandfieldnews.com/track-coach/a-better-way-tothrow-the-discus/ 
14. Ratamess, NA, Rosenberg, JG, Klei, S, Dougherty, BM, Kang, J ,Smith, CR, Ross, RE, and Faigenbaum, AD. (2015). Comparison of the acute metabolic responses to traditional resistance, bodyweight , and battling rope exercises. J Strength Cond Res 29: 47-57.

15. Ratamess, NA, Smith, CR, Beller, NA, Kang, J, Faigenbaum, AD, and Bush, JA. (2015). Effects of rest interval length on acute battling rope exercise metabolism. J Strength Cond Res 29: 2375-2387.

16. Steve Leigh, Michael T. Gross, Li \&Bing Yu (2008): The relationship between discus throwing performance and combinations of selected technical parameters Pages 173-193.

17. William.J. Bowerman: (1991): High-Performance Training for Track and Field, second edition, USA, 118. 